University of Nebraska - Lincoln

DigitalCommons@University of Nebraska - Lincoln

$1-14-2010$

\title{
Coots Use Hatch Order to Learn to Recognize and Reject Conspecific Brood Parasitic Chicks
}

Daizaburo Shizuka

University of Nebraska-Lincoln, dshizuka2@unl.edu

Bruce E. Lyon

University of California, Santa Cruz, belyon@ucsc.edu

Follow this and additional works at: https://digitalcommons.unl.edu/biosciornithology

Shizuka, Daizaburo and Lyon, Bruce E., "Coots Use Hatch Order to Learn to Recognize and Reject Conspecific Brood Parasitic Chicks" (2010). Papers in Ornithology. 86.

https://digitalcommons.unl.edu/biosciornithology/86

This Article is brought to you for free and open access by the Papers in the Biological Sciences at DigitalCommons@University of Nebraska - Lincoln. It has been accepted for inclusion in Papers in Ornithology by an authorized administrator of DigitalCommons@University of Nebraska - Lincoln. 


\title{
Coots Use Hatch Order to Learn to Recognize and Reject Conspecific Brood Parasitic Chicks
}

\author{
Daizaburo Shizuka and Bruce E. Lyon \\ Department of Ecology and Evolutionary Biology, University of California, Santa Cruz, California 95064, USA \\ Corresponding author — Daizaburo Shizuka
}

\begin{abstract}
Avian brood parasites and their hosts provide model systems for investigating links between recognition, learning, and their fitness consequences. ${ }^{I-4}$ One major evolutionary puzzle has continued to capture the attention of naturalists for centuries: why do hosts of brood parasites generally fail to recognize parasitic offspring after they have hatched from the egg, ${ }^{5-9}$ even when the host and parasitic chicks differ to almost comic degrees? ${ }^{7}$ One prominent theory to explain this pattern proposes that the costs of mistakenly learning to recognize the wrong offspring make recognition maladaptive. ${ }^{10}$ Here we show that American coots, Fulica americana, can recognize and reject parasitic chicks in their brood by using learned cues, despite the fact that the hosts and the brood parasites are of the same species. A series of chick cross-fostering experiments confirm that coots use first-hatched chicks in a brood as referents to learn to recognize their own chicks and then discriminate against later-hatched parasitic chicks in the same brood. When experimentally provided with the wrong reference chicks, coots can be induced to discriminate against their own offspring, confirming that the learning errors proposed by theory can exist. ${ }^{10}$ However, learning based on hatching order is reliable in naturally parasitized coot nests because host eggs hatch predictably ahead of parasite eggs. Conversely, a lack of reliable information may help to explain why the evolution of chick recognition is not more common in hosts of most interspecific brood parasites.
\end{abstract}

The puzzling absence of chick recognition as a host defense against avian brood parasites has fuelled a long-standing and unresolved debate over what constrains the evolution of such a seemingly obvious host adaptation, producing many alternative hypotheses. ${ }^{8,9,11-14}$ Learning is an important component of host defenses such as egg recognition, 2, 15, 16, and theory suggests that it could be fundamental to the presence or absence of chick recognition in some taxa as well. ${ }^{10,17}$ One prominent theory ${ }^{10}$ shows that if chick recognition were learned, as it is with eggs, ${ }^{2}, 15,16$ then the evolution of parasitic chick recognition in hosts of common cuckoos, Cuculus canorus, could be constrained simply by the costs of errors in learning. Shortly after hatching, cuckoo chicks evict all host eggs and chicks, ${ }^{7}$ and hosts are assumed to learn the features of their own eggs and chicks in their first breeding attempt. ${ }^{2,}$ 15 According to the theory, a host parasitized during its first breeding attempt would falsely imprint on the lone parasite as its own chick, and thereafter reject its own offspring for the rest of its life. The extreme fitness costs of such misimprinting $^{10}$ could potentially explain why so many cuckoo hosts, and perhaps the hosts of other parasitic species, ${ }^{17}$ do not recognize parasitic chicks. Because this misimprinting hypothesis was proposed to explain the absence of adaptation, it cannot be tested directly, but two corollary predictions are testable in species that do show offspring recognition: first, chick recognition can be an effective host defense when reliable information is available, and second, such recognition would involve learning. ${ }^{7,10}$ Two definitive cases of chick recognition have recently been documented; $4,13,18$ however, although previous experience honed host rejection abilities in one of these hosts, ${ }^{4}$ the learning mechanisms have yet to be elucidated. Here we confirm the corollary predictions of the misimprinting hypothesis in an unlikely system - brood parasitism within species-and describe the learning mechanism involved in chick recognition.

Conspecific brood parasitism is widespread in birds, and host defenses such as egg rejection have been documented in several taxa; however, chick recognition has rarely been examined and has never been documented. ${ }^{19}$ A previous study of American coots in British Columbia, Canada, revealed high rates of conspecific brood parasitism. ${ }^{20,21}$ Food supplied by both parents is a critical and limiting factor that affects chick survival, ${ }^{20}$ and each successful parasitic chick results in the demise of one host chick. ${ }^{22}$ These high fitness costs have favored the evolution of egg rejection as a host defense. ${ }^{21}$ We now show that hosts are also capable of recognizing and rejecting parasitic chicks, and that this recognition is learned.

An eight-year study of the survival of parasite and host chicks in naturally parasitized nests revealed that parasitic chicks suffer higher mortality than host chicks for a given hatch order (Figure 1; generalized linear mixed model with nest as random factor and controlled for hatch order: log likelihood ratio $x^{2}=8.0$, d.f. $\left.=1, P=0.005\right)$. Although this pattern is consistent with parental discrimination and rejection of

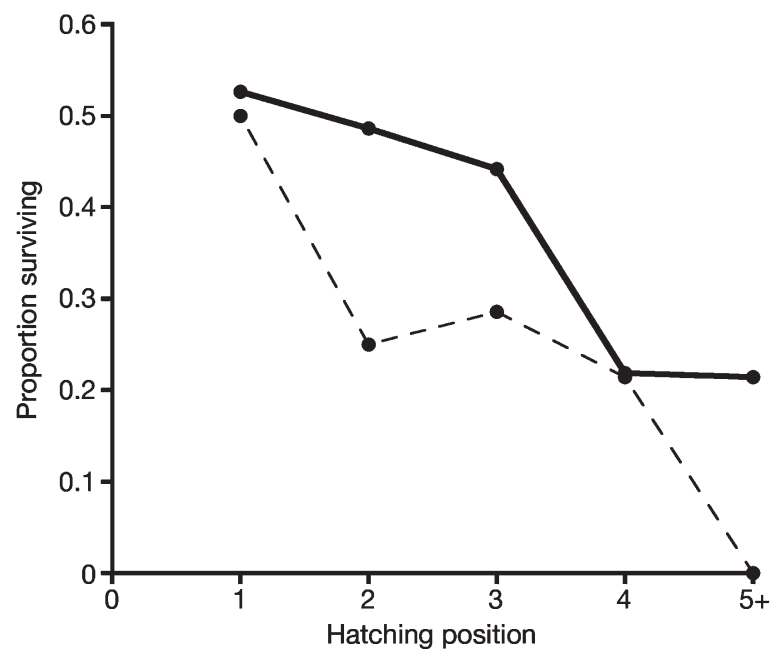

Figure I. Chick survival at naturally parasitized broods. The relationship between the hatching position of a chick (that is, the day on which the chick hatched, counting the hatching of the first chick in the brood as day I) and the proportion of host (solid line) and parasite (dashed line) chicks that survived to the end of the observation period on naturally parasitized broods ( $n=35$ broods, 203 host chicks and 62 parasitic chicks). Overall, 36\% of host chicks and $19 \%$ of parasitic chicks survived in these broods. 
Figure 2: Cross-fostering experiments to confirm chick recognition and its mechanism. a-c) Schematic representations of the design of the three recognition experiments. a, b) In "host first" and "foreign first" broods, only host chicks (white) or foreign chicks (striped), respectively, were introduced on the first day as referents. On subsequent days, each host chick was matched with a foreign chick. The actual numbers of chicks added on each day in both experiments followed natural hatching patterns and therefore varied between broods. c, In "mixed synchrony" broods, host chicks were matched with roughly equal numbers of foreign chicks on both the first and second days of hatching. All foreign chicks in each "host first" and "foreign first" brood were from the same donor clutch, but some "mixed synchrony" broods received foreign chicks from multiple donors. d-f) Results of the "host first" (d), "foreign first" (e) and "mixed synchrony" (f) experiments in terms of host chick (open circles) and foreign chick (filled circles) survival in each brood to the last census (referent chicks excluded). Dashed lines are the means of brood proportions for each chick type.

parasite chicks, we used chick cross-fostering experiments both to rule out alternative explanations for the lower survival of parasitic chicks (for example, that parasite eggs may be inherently inferior as a result of maternal effects) and to investigate how recognition occurs. Hatching chicks in incubators allowed us complete control over parental exposure to chicks and thus their access to recognition cues, and the use of nonparasitic chicks as foster chicks controlled for parasitic egg quality ${ }^{19}$ and incubation effects. ${ }^{21}$

We tested the hypothesis that coots use the first chicks that hatch in the brood as referents for recognition, following an idea suggested decades ago for egg recognition ${ }^{15}, 23$, 24 and recently supported empirically for eggs. ${ }^{16}$ Coots lay large clutches (average 9.0 eggs) that hatch very asynchronously (three to ten days for the complete clutch to hatch). At most parasitized nests, the three chicks that typically hatch on the first hatching day are all host chicks (see later). Thus, the breeding biology of coots would allow hosts to use the chicks that hatch on the first day as reliable referents for learning recognition cues that could then be applied to laterhatching chicks in the same brood. This mechanism requires no assumptions about how often birds learn their chicks and is feasible with either a single learning event during the first breeding attempt or repeated learning of recognition cues during each breeding season.

To test the hatch order hypothesis, we conducted a partial cross-fostering experiment in which on the first hatching day we gave parents only their own chicks (that is, referent chicks), and then on each subsequent hatching day we introduced an equal number of their own and foreign chicks (Figure 2a; hereafter "host first" experiment). If recognition cues are learned from the first-hatched chicks, these birds should have been able to successfully recognize their own chicks and discriminate against the foreign chicks. In support of this, a significantly higher proportion of host chicks than foreign chicks survived to independence in each brood (Figure 2d; Wilcoxon signed-rank test, $W=41.0, n=15$, one-tailed $P=0.004)$. Such highly non-random survival indicates parental discrimination in coots because food provided by parents is essential for chick survival, parents control which offspring are fed, and starvation is a major cause of chick mortality. 20,25

To rule out previous experience or innate recognition as factors that could have contributed to the outcome of the "host first" experiment, and to confirm that first-hatched chicks influence parental discrimination, we conducted a subsequent "foreign first" experiment. In this experiment, parents re- ceived only foreign chicks on the first hatching day and then equal numbers of host and foreign chicks on subsequent hatching days (Figure 2b). If parents learn their first-hatched chicks as referents, experimentally providing the wrong referents should induce learning errors and cause parents to discriminate against their own chicks. As predicted, foreign chicks had greater survival than host chicks (Figure 2e; Wilcoxon signed-rank test, $W=-32.5, n=15$, one-tailed $P=0.02$ ). It is therefore clear that the identity of first-hatched chicks (that is, host or foreign) in the brood affects the relative survival of later-hatching host and foreign chicks (generalized linear mixed model, chick type $\times$ experiment interaction; $Z=5.0$, $n=30$ broods, 251 chicks, $P<0.0001$; see full model in Supplementary Information). Experimentally induced discrimination against one's own offspring confirms that the hypothetical learning errors proposed to be fundamental to the evolution of chick recognition ${ }^{10}$ are possible. Moreover, an apparent example of misimprinting at one of our non-experimental nests indicates that some birds actually pay the fitness costs of these errors (see Supplementary Information).

One other cross-fostering manipulation, the "mixed synchrony" experiment, further confirmed that the reliability of information on the first day of hatching is critical for successful chick recognition. In this experiment, parents were given both their own chicks and foreign chicks on the first hatching day, and some broods $(n=15)$ also received a similar mix of host and foreign chicks on the second day (Figure 2c). If our hypothesis is correct, these parents should have been deprived of accurate information for learning to distinguish between their own and foreign chicks that hatched on the second day. As predicted, there was no evidence for recognition: host and foreign chicks did not differ in survival rate (Figure 2f; Wilcoxon signed-rank test on proportion of non-template chicks surviving, $W=7.0, n=15$, two-tailed $P=0.57$ ) or in parental feeding rate (Wilcoxon signed-rank test, $W=12.0, n=15$, twotailed $P=0.32$ ). The design of the "mixed synchrony" experiments also enabled us to test and reject an alternative explanation for the patterns of rejection that we observed in the "host first" and "foreign first" experiments: recognition of the majority phenotype ${ }^{26}$ (see Supplementary Information for details).

Taken together, our results provide convincing evidence that parents can recognize foreign conspecific chicks in their brood on the basis of cues learned from their first-hatched chicks. A key issue about learned chick recognition is whether or not learning is restricted to the parents' first breeding attempt. ${ }^{4}, 10$ We did not follow adults across their lives and 
Figure 3. The hatch order mechanism for chick recognition. a) A simple illustration of the key elements of the chick recognition mechanism of American coots. Three different outcomes are possible with respect to the types of chick that hatch on the first hatching day; each outcome has a different fitness consequence for hosts, so that the total fitness of the learned chick recognition strategy depends both on costs and benefits and on the frequencies of the different hatching outcomes. If only host chicks (white) hatch on the first hatching day, the host parents form the correct "host only" template and gain the benefit of correct rejection of parasitic chicks. If parasitic chicks (striped) hatch along with host chicks on the first hatching day, the host parents form a "mixed" template and accept both host and parasitic chicks. The fitness payoff for a mixed template is the same as a universal acceptor that does not recognize parasitic chicks. If only parasitic chicks hatch on the first hatching day, the host parents mistakenly learn to recognize the parasitic chicks as their own, and pay the cost of rejecting their own chicks. b) The proportion of naturally parasitized coot nests ( $n=63$ nests) that hatched only host chicks, a mix of parasite and host chicks, or only parasitic chicks on the first day of hatching.

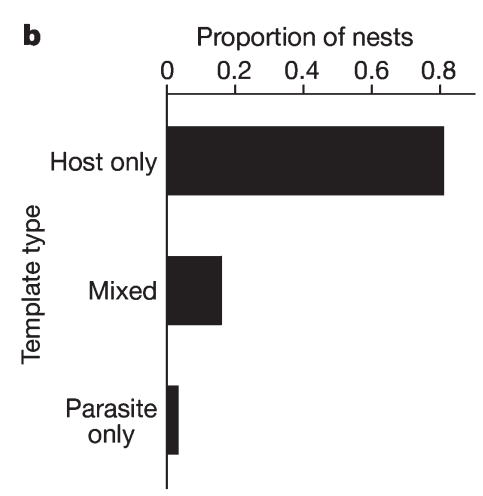

cannot directly examine how often coots use the hatch order mechanism to learn recognition cues, but our findings are not consistent with single-time imprinting unless the study population was composed almost entirely of first-time breeders, an unlikely situation (see Supplementary Information for an explanation of the patterns in a demographic context). Instead, our results suggest that recognition is based largely on templates that are relearned annually, but we do have evidence that at least some birds seem to use previously acquired templates for recognizing chicks (see Supplementary Information for details).

A critical question is how chick rejection occurs. The behavioral mechanism of chick rejection could entail either direct infanticide or more passive rejection through neglect and starvation, a behavior that is also involved in the reduction of family size in unparasitized coot broods. ${ }^{25,} 27$ The behavioral mechanisms of rejection in our experimental study were difficult to discern because the significant differences in survival between host and foreign chicks occurred very rapidly, before we could conduct our first behavioral observations at most nests (see Supplementary Information and Supplementary Figure 1). Nevertheless, at all four of the nests that we were able to watch closely early in the chick stage, we observed rejection in action. All cases involved forms and intensities of parental aggression not seen in unparasitized broods, including actively seeking the chicks from a distance to peck them vigorously and attempt to drown them, pecking chicks while brooding on the nest, and preventing chicks from access to the nest to be brooded (Supplementary Movies 1-5 and Supplementary Information). We observed similar forms of aggression only at two other nests: one case of infanticide of a natural brood parasite, and another nest in which parents killed all of their own chicks after apparently misimprinting on chicks of a neighboring pair (see Supplementary Information). These forms of parental aggression differed from the hostility that parent coots commonly use to control food allocation between surviving chicks $^{27}$ (for example, compare Supplementary Movies 1 and 3). That parental rejection was active and aggressive at all of the nests that we were able to watch sufficiently early suggests that these observations are representative of parasitic chick rejection in general in American coots.

American coots learn to distinguish their own chicks from those of conspecific brood parasites, and subsequently reduce the costs of brood parasitism by rejecting some parasitic chicks. Given that hosts of interspecific brood parasites fail to distin- guish chicks that differ in so many obvious ways from their own, the ability to recognize brood parasites of the same species seems remarkable. However, as proposed by the misimprinting hypothesis ${ }^{10}$ and bolstered by our study, the risk and costs of errors in learning-not the absolute difference in chick phenotype-may explain whether or not recognition evolves in particular taxa (Figure 3a). The use of first-hatched chicks as referents for recognition will be adaptive if the probability that parasitic chicks will hatch on the first hatching day is sufficiently low. In coots, parasites rarely hatch on the first hatching day, and this could be the key to the success of their chick recognition mechanism. Among parasitized broods in which parasitic chicks hatched, $81 \%$ of 63 clutches hatched only their own chicks on the first day and thus had reliable information, $16 \%$ hatched both types, and a mere $3 \%$ hatched only parasitic chicks on the first hatching day and could have paid the costs of misimprinting (Figure 3b). Conversely, hosts of many interspecific brood parasites would not be able to use first-hatched chicks as reliable referents because brood parasites have often evolved mechanisms to hatch early, ahead of the hosts' own chicks. ${ }^{1,28}$ However, now that we have confirmed that hatch order is used by conspecific brood parasites, it would be worth examining recognition in species in which host eggs do hatch reliably ahead of their interspecific brood parasites.

\section{Methods Summary}

We monitored and observed American coot nests on several wetlands near Williams Lake, British Columbia, from 1987 to 1990 (Reference 20) and 2005 to 2008 . We monitored each nest every one to four days during egg-laying, and we detected parasitism by the appearance of more than one new egg per day. We compared eggs visually to determine which eggs had been laid by brood parasites. ${ }^{21,} 22$ From 2005 to 2008 we hatched chicks in captivity (both natural and experimental broods), using similar methods to those in other studies on coots. ${ }^{29}$ We returned the chicks to nests within $24 \mathrm{~h}$ of hatching, after attaching color-coded nape tags ${ }^{30}$ that were unique to individuals in each brood. To control for factors that could affect the survival of parasitic chicks independently of recognition, such as parasitic egg qual$i^{1}{ }^{19}$ or discriminatory incubation, ${ }^{21}$ we did not use chicks from parasitic eggs for cross-fostering but instead used chicks from other donor nests in the population as foster chicks. For all focal broods (experimental and naturally parasitized broods), we conducted censuses and feeding observations from floating blinds, periodically visiting broods for at least 20 days after hatching, at which point chicks are no longer critically dependent on parental provisioning, ${ }^{20}$ and up to 35 days depending on the brood. We analyzed survival on the basis of the presence or absence of chicks in the brood at the last census. 


\section{Methods}

Monitoring nests and quantifying natural brood parasitism We monitored and observed nests on several wetlands near Williams Lake, British Columbia, from 1987 to 1990 (417 nests) and 2005 to 2008 (284 nests). Additional details about the study area and methods for studying brood parasitism are provided in a previously published study. ${ }^{20}$ In addition to the wetlands described previously, we worked at a complex of three wetlands along Dog Creek Road near Williams Lake (Westwick Lakes). We monitored each nest every one to four days during egg-laying (every one to two days during the 1987-1990 phase of the study), and most nests were found very early in the laying stage. On each visit, all new eggs were given a unique number with a permanent marker. We detected parasitism by the appearance of more than one new egg per day, and we compared eggs visually to determine which eggs had been laid by brood parasites. The accuracy of these methods has previously been verified by both statistical tests with egg features ${ }^{31}$ and DNA fingerprinting. ${ }^{22}$ In 1987-1990, 172 nests were parasitized, and at least one parasitic chick hatched in 61 nests. In 2005-2008, 30 nests were parasitized, and at least one parasitic chick hatched in 19 nests. The lower parasitism rate in the second phase of the study reflects two factors: focusing on different wetlands (wetlands vary in the frequency of parasitism ${ }^{20}$ ) plus a general decline over time in the frequency of parasitism. For statistical analyses of chick mortality (Figure 1), we include only the 35 nests from which we had complete census data for all host and parasitic chicks in the brood.

Hatching chicks in captivity - From 2005-2008, we hatched chicks in captivity (both control and experimental broods) to ensure that we controlled all cues available for parents to learn for chick recognition, and to also ensure that all chicks could be captured at each nest (the precocial chicks can leave the nest within hours of hatching and can thereafter be impossible to capture). Our captive hatching methods were similar to those of other studies on American coots. ${ }^{29}$ We took eggs from nests at first sign of pipping (that is, when chicks just began to break the eggshell from within), typically one or two days before the chicks hatched. We then hatched each egg inside an individual mesh pouch in an incubator (Hovabator model 1602N; GQF Manufacturing) to keep individual chicks with the eggs they hatched from; this ensured complete accuracy in the identity of the chick (that is, which chicks came from which eggs). We returned the chicks to nests within $24 \mathrm{~h}$ of hatching, after attaching color-coded nape tags ${ }^{30}$ that were unique to individuals in each brood. Because of a high degree of hatching asynchrony, nests were never left with fewer than two eggs or chicks, and parents did not abandon the nest during this period. Close monitoring of a few broods from floating blinds during and shortly after the hatching period indicated that parents readily accepted the chicks that we placed in their nests.

Monitoring broods and conducting behavioral observations We chose the study area specifically for logistic attributes that facilitate behavioral observation and census: first, high breeding densities of nesting pairs (four pairs per hectare) and small territories (mean size 0.24 ha) make it easy to find, census and observe family groups accurately; and second, on most wetlands vegetation is both sparse and restricted to a narrow band along the shoreline, making brood observations very easy. Almost all pairs spend considerable amounts of time in open water with their broods, so we were able to census broods accurately. For all focal broods (experimental and naturally parasitized broods), we conducted censuses and feeding observations periodically (average 6.3 censuses per brood) for at least 20 days, and up to 35 days, after the last chick was returned to the nest. By 20 days, chicks typically reached a size at which they could feed independently and were no longer critically dependent on parental provisioning. ${ }^{20} \mathrm{At}$ this time, mortality at control nests and experimental nests had stabilized (see Supplementary Figure 1 for survival at experimental nests). Brood censuses and behavioral observations were conducted at close range $(10-40 \mathrm{~m})$ from floating blinds equipped with camouflage coverings, from which the individually distinct nape tags could be observed easily with binoculars. Most pairs habituated very rapidly and then treated us as if we were invisible; we could therefore observe and accurately survey broods. The sex of the parent was determined by the unique vocalizations of each sex. ${ }^{32}$ Once birds had been sexed by call we noted plumage and frontal shield characteristics that enabled reliable visual identification of each of the two parents on each territory.

Of the 484 chicks observed in the experimental broods, only 18 chicks $(3.7 \%)$ ever reappeared in our censuses after not having been seen for two previous censuses. A chick was therefore considered to have survived to the end of the study period if it was observed in one of the last two censuses.
Comparisons of feeding rates were based on several focal observation periods $1 \mathrm{~h}$ long, and supplemented with opportunistic nonfocal observations. In focal observations, we focused on one parent for $30 \mathrm{~min}$, then on the second parent for the remaining $30 \mathrm{~min}$. Coots feed their chicks at a very high rate compared with that of songbirds (average 1.3 feeds per minute for the "mixed synchrony" broods used here). Broods in our analysis of feeding rates in the "mixed synchrony" experiment were observed for an average of $160 \mathrm{~min}$, with an average of 238 feeding events recorded per brood. We calculated the proportion of all feedings observed that were allocated to each chick, and then summarized these values to compare the average proportion of feeds received by host and foreign chicks. Our statistical analyses involve matched pair comparisons within broods, so the statistical comparison has great power to determine relative feeding differences between different categories of chicks. The same approach was used in a previous study of coot chick survival, and relative feeding rates between two types of chicks in broods (colorful versus trimmed) were highly significant. ${ }^{25}$ We are currently using these within-brood comparisons to study parental favoritism and again find highly significant patterns (D.S. and B.E.L., unpublished observations)

We conducted comparisons of feeding rates for "mixed synchrony" experiments only, because in "host first" and "foreign first" broods the strongly biased mortality decreased the power of the analysis and also precluded the necessity for this analysis. By the last census, only six "host first" broods had any foreign chicks alive, and only nine "foreign first" broods had any host chicks alive.

Details of experimental design and setup - All experiments involved a partial cross-fostering approach in which, in addition to including some of the chicks hatched at each focal nest, we also added chicks hatched from pipped eggs collected from non-focal "donor" nests on the study wetlands or from other nearby wetlands.

“Host first" and "foreign first" experiments - We established 15 nests per experiment in 2008, and we were able to obtain survival data for all nests. Each focal nest was matched with a donor nest that had roughly the same clutch size and began hatching on the same day. All chicks were hatched in incubators with the use of the same methods as for control broods. In the "host first" broods, we returned all host chicks that hatched on the first hatching day (range 2-6; mean 3.1 chicks). On subsequent days we matched each newly hatched host chick with a foreign chick that hatched on the same day, and returned them to the treatment nest simultaneously (range 2-7, average 4.5 pairs of chicks). If a host chick or foreign chick hatched without an appropriate match, we did not use this chick for the experiment and returned it to a non-focal nest. In "foreign first" broods, we returned foreign chicks from the donor nest on the first hatching day instead of host chicks (range 2-4, average 2.8 chicks). We then paired subsequent hatchlings (range 1-7, average 4.6 pairs) by using identical procedures to those for the "host first" treatment. Average brood sizes for "host first" and "foreign first" broods were 12.0 and 11.0 chicks, and these were not significantly different from unmanipulated broods (10.6 chicks; analysis of variance, $F=1.29$, d.f. $=2, P=0.29$ ). For statistical analyses we excluded the chicks hatched on the first day because these were "referent" chicks and were not subject to our hypothesis for chick recognition. For each brood, we pooled later-hatched chicks (that is, non-referent) and conducted matched-pair analysis on the proportion of host versus foreign chicks that survived in each brood.

“Mixed synchrony" experiment - From 2005-2007, 30 nests were set up as "mixed synchrony" nests, in which host chicks were matched with a roughly equal number of foreign chicks on the first day or first two days of hatching, depending on hatching patterns (we matched brood size to clutch size). Eggs in the focal nest were hatched in incubators as described above. On the first two days of hatching, we matched host chicks with a roughly equal number of donor chicks hatching on the same day, and we returned both types of chick at the same time. Brood size roughly matched the original clutch size ( \pm 1 chick). Foreign chicks were not necessarily from the same clutch. Chicks not included in the experimental treatment (for example, laterhatching host chicks and donor chicks) were returned to the "donor nests" or other unobserved nests. Only seven foreign chicks in these experimental nests were from natural parasitic eggs laid in a donor nest, and excluding these did not have any effect on statistical significance. The average brood size for "mixed synchrony" broods was 9.4 chicks, which was slightly smaller than for unmanipulated broods (10.6 chicks; ANOVA, $F=3.85$, d.f. $=1, P=0.06$ ). 
The "mixed synchrony" experiments were initially set up before we realized that birds might learn recognition cues each year and were thus designed to test for innate recognition or single-time learning as assumed by a previous mode ${ }^{10}$. Once we had discovered the hatching order mechanism, we were able to use a subset of the broods in that context (that is, to test for recognition in the absence of a reliable referent chick). To test the effect of first-hatched chicks as referents, we used only broods to which we returned chicks over two days and hence could test the survival of non-reference host and foreign chicks $(n=15)$. At these nests, roughly equal numbers of host and foreign chicks were returned on both the first day (range 2-8, average 4.9 chicks) and the second day (range 3-8, average 4.5 chicks) of hatching. We conducted matched-pair comparisons in the same way as in the "host first" and "foreign first" experiments, by excluding the firsthatched chicks from analyses and comparing the proportion of laterhatched host and foreign chicks that survived. We were also able to use many of these broods to test for discordancy (see Supplementary Information). In contrast to "host first" and "foreign first" broods, the foreign chicks were not necessarily siblings, which in many broods $(n$ $=21$ ) resulted in host chicks being the numerically dominant type because they outnumbered the most common foreign type. For this analysis we conducted matched-pair analysis on the proportion of all host and foreign chicks that survived in each brood.

Statistical analysis - Generalized linear mixed models were used to compare the survival of host and parasite chicks in naturally parasitized nests and to test for an interaction between the results of the "host first" and "parasite first" experimental broods. We conducted these analyses using the R software ${ }^{33}$ package lme4 (Reference 34). For the comparison of the survival of host and parasite chicks, we scored the presence or absence of chicks in the brood at the last census (see above). We then used matched-pair comparisons to compare the proportions of host and chicks that survived in each brood. We also provide an alternative method of analysis in the Supplementary Information, using a generalized linear mixed model approach. For the comparison of the "host first" and "foreign first" experiments we constructed a full model by using survival as the response variable with binomial error structure, brood identity as a random effect, and hatch order, experiment type ("host first" or "foreign first"), chick type (host or foreign), and the experiment type $\times$ chick type interaction term as fixed effects (Supplementary Table 1).

Acknowledgments - We thank J. Herrick and W. Messner for access to their property; B. Bair, L. Cargill, E. Clancey, J. Clark, J. Click, R. Drobek, S. Everding, K. Funk, J. Garcia, L. Hamilton, D. Hansen, E. Hoosier, M. Magrath, J. Melhaff, C. Morrill, C. Nelson, A. O'Brien, L. Orr, G. Peters, G. Taylor, K. Tjernell and J. Sapp for assistance in the field; B. Sinervo for advice on the study design; J. Eadie, A. Lotem, D. Mock, R. Montgomerie, R. Mulder, K. Wasson and A. Zink for helpful comments on the paper; and P. Raimondi and R. Montgomerie for statistical advice. Funding was provided by the National Geographic Society, the National Science Foundation (DDIG IOS-0808579 to D.S., and IOS 0443807 to B.E.L.), the Chapman Fund and the Sigma Xi Society. Fieldwork was conducted under permits from the Canadian Wildlife Service and the University of California, Santa Cruz, Institutional Animal Care and Use Committee. Author Shizuka designed the experiments, and developed the conceptual framework. Authors Shizuka and Lyon conducted the fieldwork and wrote the paper.

\section{Supplementary Information follows the References.}

\section{References}

I. Rothstein, S. I. A model system for coevolution-Avian brood parasitism. Annu.Rev. Ecol. Syst. 2I, 48I-508 (1990).

2. Lotem, A., Nakamura, H. \& Zahavi, A. Constraints on egg discrimination and cuckoo-host co-evolution. Anim. Behav. 49, I I85-1 209 (I995).

3. Davies, N. B. \& Welbergen, J. A. Social transmission of a host defense against cuckoo parasitism. Science 324, I 3 I8-1 320 (2009).

4. Langmore, N. E., Cockburn, A., Russell, A. F. \& Kilner, R. M. Flexible cuckoo chick-rejection rules in the superb fairy-wren. Behav. Ecol. 20, 978-984 (2009).

5. Jenner, E. Observations on the natural history of the cuckoo. By Mr. Edward Jenner. In a letter to John Hunter, Esq. F. R. S. Phil.Trans. R. Soc. 78, 219-237 (1788).
6. Dawkins, R. \& Krebs, J. R.Arms races between and within species. Proc. R. Soc. B 205, 489-5II (1979).

7. Davies, N. B. Cuckoos, Cowbirds and Other Cheats (T. \& A. D. Poyser, 2000).

8. Grim, T. The evolution of nestling discrimination by hosts of parasitic birds: Why is rejection so rare? Evol. Ecol. Res. 8, 785-802 (2006).

9. Soler, M. Co-evolutionary arms race between brood parasites and their hosts at the nestling stage. J. Avian Biol. 40, 237-240 (2009).

10. Lotem, A. Learning to recognize nestlings is maladaptive for cuckoo Cuculus canorus hosts. Nature 362, 743-745 (1993).

II. Rothstein, S. I. Successes and failures in avian egg and nestling recognition with comments on the utility of optimality reasoning. Am. Zool. 22, 547-560 ( 1982 ).

12. Davies, N. B. \& Brooke, M. D. Cuckoos versus reed warblers-Adaptations and counteradaptations. Anim. Behav. 36, 262-284 (1988).

13. Langmore, N. E., Hunt, S. \& Kilner, R. M. Escalation of a coevolutionary arms race through host rejection of brood parasitic young. $\mathrm{Na}$ ture 422, I57-160 (2003).

14. Britton, N. F., Planque, R. \& Franks, N. R. Evolution of defence portfolios in exploiter-victim systems. Bull. Math. Biol. 69, 957-988 (2007).

15. Rothstein, S. I. Mechanisms of avian egg recognition: possible learned and innate factors. Auk 91, 796-807 (1974).

16. Strausberger, B. M. \& Rothstein, S. I. Parasitic cowbirds may defeat host defense by causing rejecters to misimprint on cowbird eggs. Behav. Ecol. 20, 69l-699 (2009).

17. Lawes, M. J. \& Marthews, T. R.When will rejection of parasite nestlings by hosts of nonevicting avian brood parasites be favored? A misimprinting-equilibrium model. Behav. Ecol. I4, 757-770 (2003).

I8. Sato, N. J., Tokue, K., Noske, R. A., Mikami, O. K. \& Ueda, K. Evicting cuckoo nestlings from the nest: a new anti-parasitism behaviour. Biol. Lett. doi:I0.1098/rsbl.2009.0540 (23 September 2009).

19. Lyon, B. E. \& Eadie, J. M. A. Conspecific brood parasitism in birds: A life-history perspective. Annu. Rev. Ecol. Evol. Syst. 39, 343-363 (2008).

20. Lyon, B. E. Conspecific brood parasitism as a flexible female reproductive tactic in American coots. Anim. Behav. 46, 91 I-928 (1993).

21. Lyon, B. E. Egg recognition and counting reduce costs of avian conspecific brood parasitism. Nature 422, 495-499 (2003).

22. Lyon, B. E., Hochachka, W. M. \& Eadie, J. M. Paternity-parasitism tradeoffs: A model and test of host-parasite cooperation in an avian conspecific brood parasite. Evolution 56, I253-1266 (2002).

23. Rothstein, S. I. Mechanisms of avian egg-recognition: Additional evidence for learned components. Anim. Behav. 26, 67I-677 (1978).

24. Rodriguez-Girones, M.A. \& Lotem, A. How to detect a cuckoo egg: A signal-detection theory model for recognition and learning. Am. Nat. 153, 633-648 (1999).

25. Lyon, B. E., Eadie, J. M. \& Hamilton, L. D. Parental choice selects for ornamental plumage in American coot chicks. Nature 37I, 240-243 (1994).

26. Rothstein, S. I. Experimental and teleonomic investigation of avian brood parasitism. Condor 77, 250-27l (I975).

27. Horsfall, J. A. Brood reduction and brood division in coots. Anim. Behav. 32, $216-225$ (1984).

28. Briskie, J.V. \& Sealy, S. G. Evolution of short incubation periods in the parasitic cowbirds, Molothrus spp. Auk 107, 789-794 (1990).

29. Reed, W. L., Clark, M. E. \& Vleck, C. M. Maternal effects increase within-family variation in offspring survival. Am. Nat. 174, 685-695 (2009).

30. Foley, D. D. Use of colored markers on ducklings. N.Y. Fish Game J. 3, 240-247 (1956).

3I. Lyon, B. E. Tactics of parasitic American coots-host choice and the pattern of egg dispersion among host nests. Behav. Ecol. Sociobiol. 33, 87-I00 (I993).

32. Gullion, G.W.Voice differences between sexes in the American coot. Condor 52, 272-273 (1950).

33. R Development Core Team. R: A Language and Environment for Statistical Computing (R Foundation for Statistical Computing, 2009).

34. Bates, D. \& Maechler, M. Ime4: Linear mixed-effects models using S4 classes. R package version 0. 999375-3I; http://cran.r-project.org/ web/packages/lme4/index.html (2009). 
Supplementary Videos are attached to the html cover page of this document — http://digitalcommons.unl.edu/biosciornithology/86 — as follows:

Supplementary Video I - This movie shows a parent coot "touseling" its chick, whereby the chick is grabbed by the nape and gently shaken. This form of parental aggression is common in coot broods to control food allocation, and differs from the forms of aggression associated with infanticidal chick rejection shown in the other movies.

Supplementary Video 2 - This movie shows infanticidal parental aggression by an adult male coot towards a natural brood parasitic chick in its brood (a non-experimental brood).

Supplementary Video 3 - This movie shows an adult female coot attacking an experimental foreign chick in a Host First experimental brood. During several hours of observation the female repeatedly attacked this same chick, often actively seeking it out.

Supplementary Video 4 - This movie shows parental aggression by an adult female coot towards its own chick in a Foreign First experimental brood. The aggression towards the chicks includes pecking, pulling the chick off a floating algal mat and holding the chick's head in the water.

Supplementary Video $\mathbf{5}$ - This movie shows an adult female coot at a Foreign First Experimental nest pecking several chicks during a brooding session; all three of the chicks observed being pecked were the female's own chicks. 


\section{Supplementary Notes}

\section{GLMM analysis of experimental results}

Here, we compare the results of the Host First and Foreign First experiments with generalized linear mixed model (GLMM). We conducted these analyses using the R software ${ }^{1}$ package lme $4^{2}$. We constructed a full model using survival as the response variable with binomial error structure, brood identity as a random effect, and hatch order, experiment type (Host First or Foreign First), chick type (host or foreign), and the experiment type $\mathrm{x}$ chick type interaction term as fixed effects (Supplementary Table 1).

There was a strong effect of the experiment type $\mathrm{x}$ chick type interaction $(Z=5.0, n=30$ broods, 251 chicks, two-tailed $P<0.0001$ ), as expected from the reversed pattern of host chick versus foreign chick survival. This confirms that the types of chicks (host or foreign) presented as referents on the first day affects the non-random survival of host and foreign chicks that are introduced on subsequent days.

\begin{tabular}{ccccc}
\hline \multicolumn{5}{c}{ Supplementary Table1: Fixed-effect parameters for full GLMM model } \\
\hline & $\begin{array}{c}\text { Parameter } \\
\text { estimate }\end{array}$ & $\begin{array}{c}\text { Standard } \\
\text { Error }\end{array}$ & $Z$ & $\mathrm{P}$ \\
\hline Intercept & 2.28 & 0.52 & 4.41 & $<0.0001$ \\
\hline Chick Type (host or foreign) & -0.81 & 0.40 & -2.02 & 0.04 \\
Experiment Type (Host First or & -1.92 & 0.51 & -3.76 & $<0.001$ \\
Foreign First) & & & & $<0.0001$ \\
Hatch Order & -0.58 & 0.11 & -5.19 & $<0.0001$ \\
Chick Type x Experiment Type & 3.03 & 0.61 & 5.0 & \\
\hline
\end{tabular}

We also provide an additional separate analysis of the Host First and Foreign First experiments using a GLMM analysis, as some readers may prefer this statistical 
approach. However, this analysis is simply an alternative approach to the matched-pair analyses presented in the text. For each experiment, we constructed a full model with survival as the bivariate response variable, binomial error structure, brood as random effect, and hatch order, chick type and their interaction term as fixed effects. There was no significant interaction effect of hatch order and chick type on survival, so we eliminated this parameter from the model. We then tested the hypothesis that there was non-random mortality between host and foreign chicks for each treatment by removing chick type from the model and conducting a likelihood ratio test. As with the matchedpair comparisons, host chicks survived better than foreign chicks in Host First broods $\left(\chi^{2}\right.$ $=28.25$, d.f. $=1$, one tailed $P<0.001)$. In contrast, foreign chicks had higher survival than host chicks in Foreign First broods $\left(\chi^{2}=3.79\right.$, d.f. $=1$, one-tailed $\left.P=0.025\right)$. These results are nearly identical to those presented in the text using Wilcoxon signed-rank tests.

\section{The costs of learning to recognize: a natural case of misimprinting}

A remarkable observation in 2007 demonstrates how the costs of learning errors occur in nature. At nest 7322, we observed a pair of birds feeding two chicks from the neighboring territory on a few different days before their own chicks hatched. During a behavioral observation during the hatching stage, BEL witnessed parents vigorously pecking at their own chicks, a type of aggression seen only in experimental Host First and Foreign First broods and towards parasitic chicks in a natural brood (see below). Some chicks disappeared during the course of that observation and the rest disappeared over the course of the next few days. This observation suggested that these particular parents had learned the wrong chicks as their own and were paying the cost of this learning error. 


\section{Testing for recognition by discordancy}

By design, broods in both the Host First and Foreign First experiments had an unequal total number of host and foreign chicks (Fig. 2a,b), at least during the first few days of the experiment, so an alternative explanation for the observed survival patterns is feasible. The results of these two experiments could simply reflect parental recognition of the majority phenotype, or 'recognition by discordancy'3, because the chicks predicted and observed to have higher survival by the hatch order hypothesis in each experiment were also in the majority (Fig. 2a,b). However, four lines of evidence indicate that recognition based on the majority type, or discordancy, cannot explain our findings:

(1) Lack of recognition in experimental broods where birds could have used discordancy but not hatching order to recognize foreign chicks: By design, hatch order information was not available to birds in the Mixed Synchrony experiment, but in most broods host chicks were the majority type because the foreign chicks came from a few different nests. Thus, if birds use recognition by discordancy, foreign chicks should have suffered lower survival in broods with host chick majority, but they did not (Wilcoxon signed-rank, $W=$ 15.0, $n=21$, one-tailed $P=0.28)$.

(2) Discordancy does not predict survival of parasitic chicks in naturally parasitized nests. In naturally parasitized nests, parasitic chicks that hatched on the first hatching day survived as well as host chicks (Fig. 1 , Fisher Exact one-tailed $P=0.59$ ), whereas laterhatched parasitic chicks suffer higher mortality (pooling all later-hatched chicks, (Fisher Exact one-tailed $P=0.009)$. The hatch order mechanism predicts the absence of rejection of the first-hatched parasite chicks. Conversely, the discordancy hypothesis predicts that 
these chicks should have been recognized and rejected because parasitic chicks are always less common than host chicks at naturally parasitized nests.

(3) Two Mixed Synchrony experimental broods where hatch order but not discordancy was possible. By chance, only host chicks were introduced on the first hatching day in these two broods (they are excluded from analyses presented in the text), and a roughly equal number of foreign chicks were returned on the second day (which yielded total brood sizes of 3 host $\& 3$ foreign chicks in one nest, 4 host $\& 5$ foreign chicks in the other). All host chicks in these broods survived (7 alive, 0 dead), but all but one foreign chick died ( 1 alive, 7 dead)—a non-random pattern (two-tailed Fisher's exact test: $P=$ 0.001). Thus, recognition occurred in broods where discordancy was either not possible (first brood) or predicted an opposite pattern to what was observed (second brood).

(4) Case of natural misimprinting. The example of natural misimprinting at nest 7322, as described above, cannot be explained by discordancy because these birds rejected their own chicks despite being the majority. In fact there were no longer any of the neighboring chicks present when the parents killed off their own chicks. Instead, it seems clear that the birds misimprinted on the neighbor chicks and then rejected their own chicks once they hatched.

\section{Evidence that learning occurs annually}

The issue of when in their lifetime birds learn recognition cues is important for understanding the evolution of chick recognition as well as for interpreting the results of experiments. If birds learn through a single time imprinting ${ }^{4,5}$, then experimentally altering the referents for recognition, either with foreign chicks (Foreign First) or a mix 
of host and foreign chicks (Mixed Synchrony), would not affect the responses of older birds that would have already formed a recognition template from previous breeding attempts. With single time imprinting most birds that had bred previously would have formed correct templates in the past and would not have been fooled by our experiments. Thus, with single-time imprinting, the proportion of experienced versus first time breeders will affect the outcome of Foreign First and Mixed Synchrony experiments. We first discuss the demographic makeup of our population, and then the specific implications for our two experiments.

We determined the approximate age of a subset of adults in our study population by carefully quantifying rank leg coloration following a system that Crawford ${ }^{6}$ developed using a group of known-age coots. Though this method is not perfectly accurate (i.e., leg color is a strong but not perfect predictor of age ${ }^{6}$ ), it does enable us to determine whether the population was highly skewed towards yearlings or older adults.

We determined the age distribution for the two different phases of the study using slightly different methods. From 1988-1990 birds were captured at their nests and leg colors were described in detail from birds in the hand. Adult philopatry rates were so low ${ }^{7}$ that we were unable to collect demographic information on the same individuals across years and connect leg color information directly to demographic data. In 2008 we observed and categorized leg colors from free-ranging birds observed at close range from floating blinds. In all cases, we compared leg colors to reference color chips in the field; Crawford used Smithe's ${ }^{8}$ color system in his ageing system so we were able to match color chips to the Smithe colors described by Crawford. 
In the 1988-1990 sample, 54\% (34 of 79) of females were estimated to be at least two years old based on leg color; for males the estimate was $71 \%$ (55 of 78). In the latter phase of the study we quantified leg coloration only in 2008, the year we focused on the Host First and Foreign First experiments. In that year, we estimated that $86 \%$ of males (24 of 28) and 66\% of females (21 of 32) were older than one year. Overall, these results suggest that at least half of the breeding population were birds older than one year.

Given that more than half of our study population consists of adults older than one year, we would not expect to observe the patterns we observed in our experimental study if cues were learned only in the first breeding attempt. For example, if experienced breeders had learned recognition cues from their previous breeding attempt, we would have detected some discrimination in the Mixed Synchrony broods. No discrimination was observed. With similar logic, older birds in the Foreign First experiment should not have been fooled by the foreign chicks on the first hatching day, and their correct recognition should have canceled out the incorrect recognition we induced in young, first time breeders, effectively predicting no evidence for recognition. Contrary to this prediction, we observed clear evidence of recognition.

On the other hand, there is evidence that some birds may use previously acquired cues for recognition. If templates were only learned every year, we would expect no differences between the Host First and Foreign First experiments in the survival of reference chicks. However, we found that reference chicks in Foreign First broods suffered higher mortality than those in Host First broods (Restricted Maximum Likelihood, with nest as random factor: $F=6.03$, d.f. $=1, P=0.02$ ), suggesting that parents were less likely to accept foreign chicks as referents. This suggests that some parents had prior information 
on cues that reliably identify their own chicks and were less likely to accept foreign chicks as referents compared to their own chicks. The difference between the two experiments is expected because in the Foreign First experiment predictions for previous breeders and first time breeders are in opposite directions, whereas in the Host First experiment, predictions for previous and naïve breeders are in the same direction-both will favor host chicks over foreign chicks. Together, these observations suggest that recognition is largely based on templates that are completely relearned annually, but at least some birds appear to use previously acquired templates for recognizing chicks.

\section{Evidence that rejection occurs early in parental care stage}

The ease of behavioral observations at coot nests can vary across individuals, vegetation density, and time. American coot chicks are able to leave the nest for periods of time within a day of hatching, but rarely do so for the first several days. Throughout the 3 to 10 day hatching period, and for several days after, parents often feed the young in the nest, or very close to it, and most nests are hidden back in vegetation, making observations difficult. Therefore, our ability to conduct accurate censuses and feeding observations were often very limited during the first days of parental provisioning. In Host First broods, the timing of first census ranged from 1-13 days after the last hatching day (median 4 days). In Foreign First broods, first censuses were conducted 1-10 days after last hatching day (median 3 days). A significant proportion of the total mortality observed occurred before our first census (74\% for Host First broods, and 43\% for Foreign First broods). As a result, biased mortality of foreign chicks was already apparent in Host First broods at the first census (Wilcoxon signed-rank, $W=38.5, n=15$, onetailed $P=0.002$ ) and there was a trend for more host chicks to have died compared to 
foreign chicks by the first census in Foreign First broods (Wilcoxon signed-rank, $W=$ 11.5, $n=15$, one-tailed $P=0.059$ ). It is also clear that beyond 10 days after the last hatching day, mortality is less biased, as template, host and foreign chicks all suffer similarly low level of mortality (Supplementary Fig. 1). Overall, the combination of rapid mortality and difficulty in observing broods early in the chick stage meant that we had detailed early behavioral observations of the process of chick rejection for very few nests.

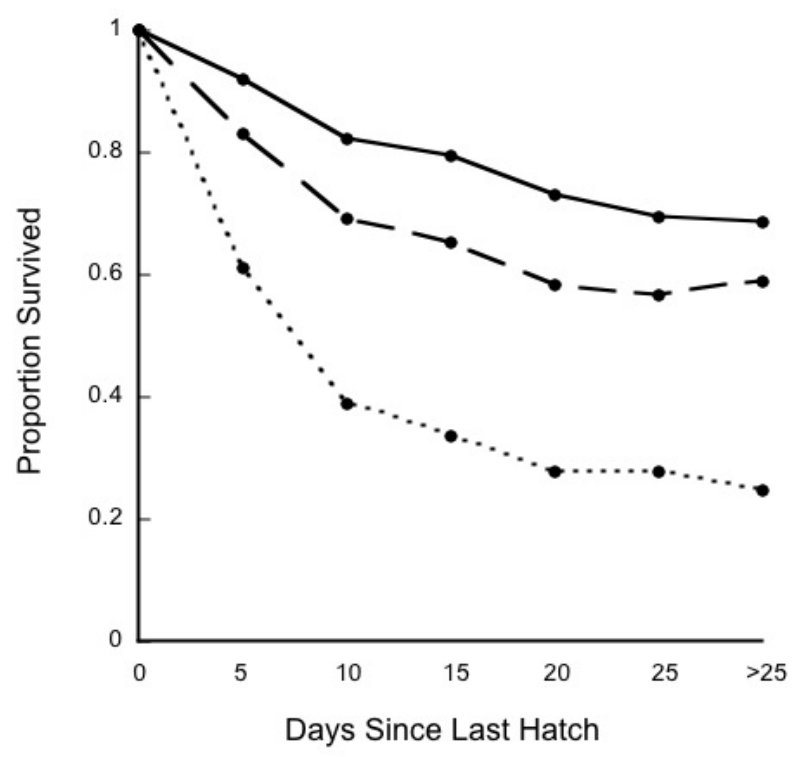

\section{Supplementary Figure 1: Comparison of survival in Host First and} Foreign First experiments. Lines show the proportions of "template" chicks (solid line), "in” chicks (i.e. host chicks in Host First broods and foreign chicks in Foreign First broods, dashed line), and "out" chicks (i.e. foreign chicks in Host First broods and host chicks in Foreign First broods, dotted line) across census periods during parental care.

\section{Additional anecdotal evidence for chick recognition and rejection}

Two observations not mentioned in the text add anecdotal evidence that chick recognition and rejection occurs in a natural context: a nest where a pair adopted back their own 
chicks that had been laid as parasitic eggs in a neighbor's nest and a nest where parents killed a brood parasitic chick.

Adoption of own chicks laid as parasitic eggs in neighboring nest: On June 16, 2005, D.S. observed a rare case of adoption at Nest 5009. Three pairs of chicks with the same band combinations were observed (orange-orange, white-white and blue-blue), despite our records showing only one chick of each of these combinations were returned to the nest. Our records also indicated that between June 11 and June 13, three chicks with these same tag combinations disappeared from Nest 5026 on the adjacent territory. Therefore, we can be confident that these three chicks from Nest 5026 were adopted by parents at Nest 5009. Retrospective comparisons of eggs and hatching data indicated that these three adopted chicks hatched from eggs that were among the four eggs that female 5009 had laid parasitically in Nest 5026, prior to laying eggs in her own nest. Therefore, these three chicks were parasitic chicks that were simply 'adopted’ back by their biological parents. Given that adoption is very rare in our population, the adoption of three chicks back by biological parents suggests that chick recognition was involved-even though these parents had no previous experience with the adopted chicks, the chicks presumably had the same recognition cues as the adopting parents' other chicks. The alternative explanation, that the adoption of three biological offspring was a chance event, can be ruled out. Given the fraction of host $(n=10)$ and parasitic chicks $(n=4)$ in the donor nest, the probability that all three adopted chicks were the genetic offspring of the adopting female was non-random (randomization test, $P=0.01,10000$ runs).

Infanticide of natural brood parasitic chick: On June 12, 2005, B.E.L. observed the first definitive instance of infanticide of an offspring in this population (documented in 
Supplemental Movie 2) at Nest 5012 at Jaimeson Meadow. The chick was one of two parasitic chicks present in the brood of eight chicks, and was 4 days old at the time of observation. All six of the host chicks hatched before the two parasitic chicks. Both parents were involved in extreme parental aggression toward the parasitic chick, often chasing it even when it was well separated from the rest of the brood. The parents appeared to respond particularly strongly in response to distress calls of the chick. The chick was last seen swimming towards shore, outside of territory borders and D.S. found the chick dead on shore a few hours later. The identity of the chick was confirmed by both the nape tag color and the brood number written in permanent marker on the tag (we write brood numbers on tags to keep chick identities clear when setting up experiments but these numbers typically disappear over time).

Parental behaviors involved in the infanticide of this parasitic chick in 2005 share many common features with observations of chick rejection at one control nest in 2007, where parents appeared to mistakenly learn the neighbor's chicks as their own (see S5 above), and at three experimental broods in 2008 (documented in Supplemental Movies 2-4). In contrast to "normal” tousling behavior seen in both European coots, Fulica atra ${ }^{9}$ and American coots (Shizuka and Lyon unpublished manuscript, Supplemental Movie 1) these instances of infanticidal behavior included intense pecking, holding the chicks under water, and dragging the chicks by the head. In addition, parents were observed to actively seek out targeted chicks from up to $10 \mathrm{~m}$ away. The fact that we observed similar behaviors in both natural instances as well as experimental broods suggest that active aggression is likely to be involved in the process of parasitic chick rejection. However, infanticide may not be unique to rejection of parasitic chicks, as a previous study on 
European coots suggested that parental infanticide may also be used in the context of brood reduction, where parents reduce their brood size by killing off some of their own chicks $^{9}$.

\section{References}

1. R Development Core Team. R: A language and environment for statistical computing. R Foundation for Statistical Computing, Vienna, Austria. ISBN 3-900051-07-0, URL http://www.R-project.org. (2009).

2. Bates, D., Maechler, M. lme4: Linear mixed-effects models using S4 classes. R package version 0.999375-31. http://CRAN.R-project.org/package=lme4 (2009).

3. Rothstein, S. I. Experimental and teleonomic investigation of avian brood parasitism. Condor 77, 250-271 (1975).

4 Lotem, A. Learning to recognize nestlings is maladaptive for cuckoo Cuculus-canorus hosts. Nature 362, 743-745 (1993).

5. Lotem, A., Nakamura, H. \& Zahavi, A. Constraints on egg discrimination and cuckoohost co-evolution. Anim. Behav. 49, 1185-1209 (1995).

6. Crawford, R. D. Tarsal color of American coots in relation to age. Wilson Bull. 90, 536-543 (1978).

7. Lyon, B. E. The Ecology and Evolution of Conspecific Brood Parasitism in American Coots (Fulica americana). Thesis, Princeton University, Princeton N.J. (1992).

8. Smithe, F. B. Naturalist's color guide. American Museum of Natural History, New York (1975).

9. Horsfall, J. A. Brood reduction and brood division in coots. Anim. Behav. 32, 216-225 (1984). 